\title{
WELL-POSEDNESS OF AN INVERSE PROBLEM OF A TIME-DEPENDENT GINZBURG-LANDAU MODEL FOR SUPERCONDUCTIVITY*
}

\author{
JISHAN FAN ${ }^{\dagger}$ AND SONG JIANG ${ }^{\ddagger}$
}

\begin{abstract}
In this paper we prove the existence and uniqueness of solutions to an inverse problem of a time-dependent Ginzburg-Landau model for superconductivity in the case of integral overdetermination.
\end{abstract}

Key words. Ginzburg-Landau model, superconductivity, inverse problem, integral overdetermination.

AMS subject classifications. 49K20, 35K55, 82D55

\section{Introduction}

We shall study the following Ginzburg-Landau equations for superconductivity:

$$
\begin{aligned}
& \eta \psi_{t}+i \eta k \phi \psi+\left(\frac{i}{k} \nabla+A\right)^{2} \psi+\left(|\psi|^{2}-1\right) \psi=0, \\
& A_{t}+\nabla \phi+\operatorname{curl}^{2} A+\operatorname{Re}\left\{\left(\frac{i}{k} \nabla \psi+\psi A\right) \bar{\psi}\right\}=\operatorname{curl} H \text { in } \Omega \times(0, T),
\end{aligned}
$$

with boundary and initial conditions

$$
\begin{aligned}
& \nabla \psi \cdot n=0, A \cdot n=0, \operatorname{curl} A \times n=H \times n \text { on } \partial \Omega \times(0, T), \\
& \psi(\cdot, 0)=\psi_{0}, A(\cdot, 0)=A_{0} \text { in } \Omega
\end{aligned}
$$

and the condition of integral overdetermination

$$
\int_{\Omega} A_{t}(x, t) w(x) d x=g^{\prime}(t), \quad 0 \leq t \leq T
$$

Here $\Omega \subseteq \mathbb{R}^{2}$ is a bounded domain with smooth boundary $\partial \Omega, n$ is the unit outward normal vector of $\partial \Omega ; \psi \in \mathbb{C}, A \in \mathbb{R}^{2}$ and $\phi \in \mathbb{R}$ denote the order parameter, the magnetic potential and the electric potential, respectively; $H:=f(t) h(x, t)$ is the applied magnetic field where the vector $h(x, t) \in \mathbb{R}^{2}$ is known in advance, while the unknown scalar coefficient $f \in \mathbb{R}$ is sought (here we may interpret $h$ as the applied magnetic field, while $f$ is just the scalar coefficient.); $\eta$ and $k$ are the Ginzburg-Landau positive constants; $i=\sqrt{-1}, \bar{\psi}$ denotes the complex conjugate of $\psi, \operatorname{Re} \psi=(\psi+\bar{\psi}) / 2,|\psi|^{2}=\psi \bar{\psi}$ is the density of superconducting carriers, $T$ is a given positive number. $w(x) \in \mathbb{R}^{2}$ and $g(t) \in \mathbb{R}$ are known functions. In (1.1)-(1.5), the unknown functions are $\psi, A, \phi$ and $f(t)$.

*Received: January 29, 2005; accepted (in revised version): June 25, 2005. Communicated by Jack Xin.

Supported by NSFC (Grant No. 10301014, 10225105) and the CAEP (Grant No. 2003-R-02).

†Department of Mathematics, Suzhou University, Suzhou 215006, P. R. China, College of Information Sciences and Technology, Nanjing Forestry University, Nanjing 210037, P. R. China (fanjishan@njfu.edu.cn).

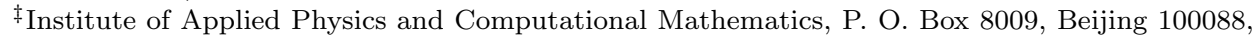
P. R. China (jiang@iapcm.ac.cn).

Dedicated to Professor Lishang Jiang on the occasion of his 70th birthday. 
For the reader's convenience, we give the definition of a curl operator:

$$
\operatorname{curl} A:=\frac{\partial A_{2}}{\partial x}-\frac{\partial A_{1}}{\partial y} \operatorname{andcurl} \phi:=\left(\frac{\partial \phi}{\partial y},-\frac{\partial \phi}{\partial x}\right)
$$

when $A \in \mathbb{R}^{2}$ and $\phi \in \mathbb{R}$ in a two dimensional domain.

It is well-known that the Ginzburg-Landau equations are gauge invariant, that is, if $(\psi, A, \phi)$ is a solution of (1.1)-(1.4), then there exists a function $\chi$ such that $\left(\psi e^{i k \chi}, A+\nabla \chi, \phi-\chi_{t}\right)$ is also a solution of (1.1)-(1.4). So, in order to obtain the wellposedness of the problem, we need to impose the gauge condition. From the physical point of view, one usually has three types of the gauge condition:

1. Coulomb gauge: $\operatorname{div} A=0$ in $\Omega, \int_{\Omega} \phi d x=0$

2. Lorentz gauge: $\phi=-\operatorname{div} A$ in $\Omega$

3. Temporal gauge: $\phi=0$ in $\Omega$

For initial data $\psi_{0} \in H^{1}(\Omega) \cap L^{\infty}(\Omega), A_{0} \in H^{1}(\Omega)$, Chen, Elliott, Tang and Du $[1,2,10,4]$ proved the existence and uniqueness of global strong solutions to (1.1)(1.4) in the case of the Coulomb and Lorentz as well as Temporal gauges.

In 1995, Tang and Wang [11] studied the Coulomb gauge case and proved the existence of global weak solutions with initial data $\left(\psi_{0}, A_{0}\right) \in L^{2}(\Omega) \times L^{2}(\Omega)$ in the two dimensional case, and Fan [5] showed the existence and uniqueness of global weak solutions and the existence of the maximal and exponential attractors with $\left(\psi_{0}, A_{0}\right) \in$ $L^{2}(\Omega) \times L^{2}(\Omega)$ in the case of the Lorentz gauge. Recently, Fan and Jiang [6] proved the existence of global weak solutions when $\Omega \subseteq \mathbb{R}^{3}$ and $\left(\psi_{0}, A_{0}\right) \in L^{2}(\Omega) \times L^{2}(\Omega)$ in the case of Coulomb gauge or Lorentz gauge, which answers an open problem in [11].

In this paper, our aim is to study the nonlinear inverse problem which consists of finding a set of the functions $\{\psi, A, \phi, f\}$ satisfying (1.1)-(1.5) which is related to the optimal control problem studied by Z.M.Chen and K.H.Hoffmann [3]. However, to our best knowledge, there is no article in the literature which investigates inverse problems of (1.1)-(1.5). We shall use the contraction mapping principle to prove our result, Theorem 1.3. The difficulty in the proof is to show that the nonlinear operator maps a bounded closed convex set into itself in a suitable space, and thus, this requires that $T$ must be small enough. Unfortunately, we are not able to give an existence result with large $T$ for general data here.

To study the inverse problem of (1.1)-(1.5), we assume throughout this paper that

(H1) $\psi_{0} \in H^{1}(\Omega),\left|\psi_{0}\right| \leq 1, A_{0} \in L^{2}(\Omega), A_{0} \cdot n=0$ on $\partial \Omega$;

(H2) $h(x, t) \in L^{\infty}\left(0, T ; L^{2}(\Omega)\right)$;

(H3) $w(x) \in H^{2}(\Omega), \operatorname{div} w=0$ in $\Omega, w \cdot n=0$ and $\operatorname{curl} w \times n=0$ on $\partial \Omega$;

(H4) $g(t) \in H^{1}(0, T)$;

(H5) $\int_{\Omega} h(x, t) \operatorname{curl} w d x=h_{0}(t) \in L^{\infty}(0, T)$ with $\left|h_{0}(t)\right| \geq h(T)>0$.

First, we state an existence and uniqueness result to the direct problem (1.1)-(1.4). 
Theorem 1.1. Let (H1)-(H2) be satisfied and $f(t) \in L^{2}(0, T)$. Then, for the Coulomb gauge, there exists a unique solution $(\psi, A, \phi)$ satisfying

$$
\begin{aligned}
& \psi \in L^{\infty}\left(0, T ; H^{1}(\Omega)\right) \cap L^{2}\left(0, T ; H^{2}(\Omega)\right), \psi_{t} \in L^{2}(\Omega \times(0, T)),|\psi| \leq 1 \text { in } \Omega \times(0, T), \\
& A \in L^{\infty}\left(0, T ; L^{2}(\Omega)\right) \cap L^{2}\left(0, T ; H^{1}(\Omega)\right), A_{t} \in L^{2}\left(0, T ; H^{-1}(\Omega)\right), \\
& \operatorname{div} A=0 \text { in } \Omega \times(0, T), \phi \in L^{2}\left(0, T ; H^{1}(\Omega)\right) .
\end{aligned}
$$

REMARK 1.1. When $\left(\psi_{0}, A_{0}\right) \in L^{2}(\Omega) \times L^{2}(\Omega)$, the existence is proved in [11], while the uniqueness is obtained in [7]. Hence, the proof of Theorem 1.1 is omitted here. Next, we derive necessary a priori estimates and give a stability result with respect to $f(t)$ which will be used in the proof of the main result in Section 3.

Based on Theorem 1.1, we can define the nonlinear operator

$$
B: L^{2}(0, T) \rightarrow L^{2}(0, T)
$$

acting in accordance with the rule

$$
(B f)(t):=\frac{1}{h_{0}}\left\{g^{\prime}(t)+\int_{\Omega}\left[A \operatorname{curl}^{2} w+w \operatorname{Re}\left(\frac{i}{k} \nabla \psi+\psi A\right) \bar{\psi}\right] d x\right\},
$$

where $(\psi, A)$ has been already found as the unique solution of the system (1.1)-(1.4).

We proceed to study the operator equation of the second kind over the space $L^{2}(0, T)$ :

$$
f=B f
$$

An interrelation between the inverse problem (1.1)-(1.5) and the nonlinear equation (1.6) from the viewpoint of their solvability is revealed in the following assertion.

THEOREM 1.2. Let (H1)-(H5) be satisfied, then the inverse problem (1.1)-(1.5) is solvable if and only if the equation (1.6) has a solution.

Proof. The proof is the same as that of [9, pp.257-259], however, for the reader's convenience, we present the proof.

We first prove the if part. Let the inverse problem (1.1)-(1.5) possess a solution, say $\{\psi, A, \phi, f\}$. Now multiplying (1.2) by $w$ and integrating by parts we arrive at

$$
\begin{aligned}
& \int_{\Omega} A_{t} w d x+\int_{\Omega} w \nabla \phi d x+\int_{\Omega} w \operatorname{curl}^{2} A d x+\int_{\Omega} w \operatorname{Re}\left(\frac{i}{k} \nabla \psi+\psi A\right) \bar{\psi} d x \\
= & f(t) \int_{\Omega} w \operatorname{curl} h d x
\end{aligned}
$$

using (H3) we know that

$$
\int_{\Omega} w \nabla \phi d x=0
$$

$$
\int_{\Omega} \operatorname{curl}(\operatorname{curl} A-f(t) h) \cdot w d x=\int_{\Omega}(\operatorname{curl} A-f(t) h) \operatorname{curl} w d x=\int_{\Omega} A \operatorname{curl}^{2} w d x-f(t) h_{0}(t) .
$$


Inserting (1.8)(1.9) into (1.7) and using (1.5) we conclude that $f$ solves the equation (1.6).

We prove the only if part. We suppose that equation (1.6) possesses a solution, say $f \in L^{2}(0, T)$. By Theorem 1.1 on the unique solvability of the direct problem we are able to recover $\{\psi, A, \phi\}$ as the solution of (1.1)-(1.4) associated with $f$, so that it remains to be shown that the function $A$ satisfies the overdetermination condition (1.5), which follows from (1.6)(1.7)(1.8)(1.9) immediately. This provides support for the view that $\{\psi, A, \phi, f\}$ is just a solution of the inverse problem (1.1)-(1.5).

REMARK 1.2. In [9], the condition of integral overdetermination is given by

$$
\int_{\Omega} A(x, t) w(x) d x=g(t), \int_{\Omega} A_{0}(x) w(x) d x=g(0)
$$

Here instead of (1.10), we use (1.5) as the condition of integral overdetermination. The reason is that in our opinion the condition (1.5) is easier to be measured in practice. Moreover, it is not difficult to see from the proof that Theorem 1.2 still holds when (1.5) is replaced by (1.10).

Now we are in a position to state our MAIN THEOREM:

THEOREM 1.3. Let (H1)-(H5) be satisfied and $T$ be small enough, then, there exists a unique solution $\{\psi, A, \phi, f\}$ to the inverse problem (1.1)-(1.5) with $f(t) \in D$, where

$$
D:=\left\{f(t) \in L^{2}(0, T) ;\left\|f-\frac{g^{\prime}}{h_{0}}\right\| \leq R\right\}
$$

and $R$ is a positive constant determined in Lemma 3.1 in Section 3.

REMARK 1.3. i) The conditions $\operatorname{div} w=0$ in $\Omega, w \cdot n=0$ on $\partial \Omega$ in (H3) do not need if $\psi_{0} \in H^{2}(\Omega)$ with some tedious calculations, we do not consider this problem for simplicity.

ii) When $h(x, t)=\operatorname{curl} w, \operatorname{curl} A \times n=h \times n=0$. We can easily prove that $T$ could be taken $T=+\infty$ in Theorem 1.3. However, it is difficult to generalize this global result. iii) In the three-dimensional case, i.e., $\Omega \subset \mathbb{R}^{3}$, if, in addition, we assume $\psi_{0} \in$ $H^{1}(\Omega) \cap L^{\infty}(\Omega), A_{0} \in H^{1}(\Omega)$ and study the equation (1.6) in $H^{1}(0, T)$. Then, we can obtain the same result. However, for simplicity, we treat here only the two-dimensional case.

iv) If one chooses the Lorentz or Temporal gauge, the same result can be obtained without essential changes in arguments.

In Section 2, we give some preliminaries to the proof of Theorem 1.3 which is given in Section 3.

\section{Preliminaries}

In this section we derive necessary estimates which are needed in the proof of Theorem 1.3.

\section{LEMMA 2.1.}

$$
\begin{aligned}
\frac{\eta}{2} \int_{\Omega} \psi^{2} d x & +\int_{0}^{T} \int_{\Omega}\left|\frac{i}{k} \nabla \psi+\psi A\right|^{2} d x+\int_{0}^{T} \int_{\Omega}\left(|\psi|^{2}-1\right)^{2} d x d t+\int_{0}^{T} \int_{\Omega}|\psi|^{2} d x d t \\
= & \frac{\eta}{2} \int_{\Omega}\left|\psi_{0}\right|^{2} d x+|\Omega| T,
\end{aligned}
$$

$|\psi| \leq 1 \quad$ in $\quad \Omega \times(0, T)$. 
Proof. Multiplying (1.1) by $\bar{\psi}$ and integrating over $\Omega \times(0, T)$, and then taking the real part on both sides of the resulting equation, we obtain (2.1). The proof of $(2.2)$ can be found in $[1,2,10,4]$.

In the proof of the following lemmas, we will repeatedly use the following inequality $[8]$ :

$$
\|u\| \leq c_{1}(\|\operatorname{div} u\|+\|\operatorname{curl} u\|)
$$

for $u \in H_{n}^{1}(\Omega):=\left\{u \in H^{1}(\Omega) \mid u \cdot n=0 \quad\right.$ on $\left.\quad \partial \Omega\right\}$.

LEMMA 2.2 .

$$
\begin{aligned}
& \int_{\Omega} A^{2} d x+\int_{0}^{T} \int_{\Omega}|\operatorname{curl} A|^{2} d x d t \\
& \leq \int_{\Omega} A_{0}^{2} d x+2 c_{1}^{2} \int_{0}^{T} \int_{\Omega}\left|\frac{i}{k} \nabla \psi+\psi A\right|^{2} d x d t+2\|h\|_{L^{\infty}\left(0, T ; L^{2}(\Omega)\right)}^{2} \int_{0}^{T} f^{2} d t .
\end{aligned}
$$

Proof. Multiplying (1.2) by $A$ in $L^{2}(\Omega)$ and integrating by parts, we have

$$
\begin{aligned}
\frac{1}{2} \frac{d}{d t} \int_{\Omega} & A^{2} d x+\int_{\Omega}|\operatorname{curl} A|^{2} d x \\
& \leq \int_{\Omega}\left|\frac{i}{k} \nabla \psi+\psi A\right||A| d x+\int_{\Omega}|\operatorname{curl} A||f h| d x \\
& \leq c_{1}\left\|\frac{i}{k} \nabla \psi+\psi A\right\|\|\operatorname{curl} A\|+\|\operatorname{curl} A\|\|f h\| \\
& \leq \frac{1}{2}\|\operatorname{curl} A\|^{2}+c_{1}^{2}\left\|\frac{i}{k} \nabla \psi+\psi A\right\|^{2}+\|f h\|^{2} .
\end{aligned}
$$

Integration of the above inequality gives (2.4).

The following lemma gives an estimate on $\nabla \phi$ and can be found in [6].

LEMMA 2.3. $\phi$ satisfies

$$
\begin{aligned}
& -\Delta \phi=\operatorname{div} \operatorname{Re}\left\{\left(\frac{i}{k} \nabla \psi+\psi A\right) \bar{\psi}\right\} \quad \text { in } \quad \Omega \times(0, T), \\
& \nabla \phi \cdot n=0 \text { on } \partial \Omega \times(0, T) .
\end{aligned}
$$

Moreover,

$$
\|\nabla \phi\|_{L^{2}(\Omega \times(0, T))} \leq\left\|\frac{i}{k} \nabla \psi+\psi A\right\|_{L^{2}(\Omega \times(0, T))} .
$$

In the calculations follow, the following Gagliardo-Nirenberg inequalities will frequently be used:

$$
\begin{aligned}
& \|A\|_{L^{4}(\Omega)} \leq C_{2}\|A\|^{1 / 2}\|\operatorname{curl} A\|^{1 / 2}, \\
& \|\nabla \psi\|_{L^{4}(\Omega)} \leq C_{3}\|\nabla \psi\|^{1 / 2}\|\Delta \psi\|^{1 / 2} .
\end{aligned}
$$


We proceed to derive bounds on derivatives of $\psi$.

Lemma 2.4. There exist positive constants $C_{4}$ and $C_{5}$, such that

$$
\begin{aligned}
\|\nabla \psi\|_{L^{\infty}\left(0, T ; L^{2}(\Omega)\right)}^{2} & \leq C_{4}\left(1+\|f\|^{4}\right) \exp \left(C_{5}\|f\|^{2}\right), \\
\|\triangle \psi\|_{L^{2}(\Omega \times(0, T))}^{2} & \leq C_{4}\left(1+\|f\|^{8}\right) \exp \left(C_{5}\|f\|^{2}\right) .
\end{aligned}
$$

Proof. Multiplying (1.1) by $-\triangle \bar{\psi}$ in $L^{2}$ and integrating by parts, taking then the real part, we find that

$$
\begin{aligned}
& \frac{\eta}{2} \frac{d}{d t} \int_{\Omega}|\nabla \psi|^{2} d x+\frac{1}{k^{2}} \int_{\Omega}|\triangle \psi|^{2} d x \\
& \leq \eta k\|\nabla \phi\|\|\nabla \psi\|+\frac{2}{k}\|A\|_{L^{4}}\|\nabla \psi\|_{L^{4}}\|\triangle \psi\|+\|A\|_{L^{4}}^{2}\|\triangle \psi\|+2\|\nabla \psi\|^{2} \\
& \leq \eta k\|\nabla \phi\| \nabla \psi\left\|+\frac{2}{k} C_{2} C_{3}\right\| A\left\|^{1 / 2}\right\| \operatorname{curl} A\left\|^{1 / 2}\right\| \nabla \psi\left\|^{1 / 2}\right\| \triangle \psi \|^{3 / 2} \\
& \quad+C_{2}^{2}\|A\|\|\operatorname{curl} A\|\|\triangle \psi\|+2\|\nabla \psi\|^{2} \\
& \leq \frac{1}{2 k^{2}}\|\triangle \psi\|^{2}+\eta k\|\nabla \phi\|\|\nabla \psi\|+2^{8} k^{2} C_{2}^{4} C_{3}^{4}\|A\|^{2}\|\operatorname{curl} A\|^{2}\|\nabla \psi\|^{2} \\
& \quad+k^{2} C_{2}^{2}\|A\|^{2}\|\operatorname{curl} A\|^{2}+2\|\nabla \psi\|^{2},
\end{aligned}
$$

which gives

$$
\begin{aligned}
& \frac{d}{d t} \int_{\Omega}|\nabla \psi|^{2} d x+\frac{1}{\eta k^{2}} \int_{\Omega}|\triangle \psi|^{2} d x \leq\left(\frac{k}{2}+\frac{2}{\eta}+\frac{1}{\eta} 2^{8} k^{2} C_{2}^{4} C_{3}^{4}\|A\|^{2}\|\operatorname{curl} A\|^{2}\right)\|\nabla \psi\|^{2} \\
& \quad+\frac{k}{2}\|\nabla \phi\|^{2}+\frac{k^{2} C_{2}^{2}}{\eta}\|A\|^{2}\|\operatorname{curl} A\|^{2} .
\end{aligned}
$$

Applying Gronwall's inequality to the above inequality, one infers

$$
\begin{aligned}
& \|\nabla \psi\|^{2} \leq\left(\left\|\nabla \psi_{0}\right\|^{2}+\frac{k}{2}\left\|\frac{i}{k} \nabla \psi+\psi A\right\|_{L^{2}(\Omega \times(0, T))}^{2}+\frac{k^{2} C_{2}^{2}}{\eta}\|A\|^{2} \int_{0}^{T}\|\operatorname{curl} A\|^{2} d t\right) \\
& \times \exp \left(\frac{k}{2} T+\frac{2}{\eta} T+\frac{1}{\eta} 2^{8} k^{2} C_{2}^{4} C_{3}^{4}\|A\|^{2} \int_{0}^{T}\|\operatorname{curl} A\|^{2} d t\right) \\
& \leq\left\{\left\|\nabla \psi_{0}\right\|^{2}+\frac{k}{2}\left(\frac{\eta}{2}\left\|\psi_{0}\right\|^{2}+|\Omega| T\right)\right. \\
& \left.+\frac{k^{2} C_{2}^{2}}{\eta}\left[\left\|A_{0}\right\|^{2}+2 C_{1}^{2}\left(\frac{\eta}{2}\left\|\psi_{0}\right\|^{2}+|\Omega| T\right)+2 \int_{0}^{T} f^{2} d t\|h\|^{2}\right]^{2}\right\} \\
& \times \exp \left[\frac{k}{2} T+\frac{2}{\eta} T+\frac{1}{\eta} 2^{8} k^{2} C_{2}^{4} C_{3}^{4}\left(\left\|A_{0}\right\|^{2}+2 C_{1}^{2}\left(\frac{\eta}{2}\left\|\psi_{0}\right\|^{2}+|\Omega| T\right)+2 \int_{0}^{T} f^{2} d t\|h\|^{2}\right)\right] \\
& \leq C_{4}\left(1+\|f\|^{4}\right) \exp \left(C_{5}\|f\|^{2}\right),
\end{aligned}
$$

which leads to (2.10).

Integrating $(2.12)$ over $(0, T)$, we obtain (2.11). This completes the proof.

The following lemma is concerned with the continuous dependence of solutions on data and will be used in the proof of the contraction property of the operator $B$. 
Lemma 2.5. Let $\left(\psi_{i}, A_{i}, \phi_{i}\right)(i=1,2)$ be the corresponding solutions of the problem (1.1)-(1.4) with the data $f_{i}(i=1,2)$, respectively. Then, there exists a positive constant $C_{6}$, such that

$$
\begin{aligned}
& \left\|\psi_{1}-\psi_{2}\right\|_{L^{\infty}\left(0, t ; L^{2}(\Omega)\right)}^{2}+\left\|A_{1}-A_{2}\right\|_{L^{\infty}\left(0, t ; L^{2}(\Omega)\right)}^{2} \\
& \quad \leq \int_{0}^{t}\left|f_{1}(\tau)-f_{2}(\tau)\right|^{2} d \tau \exp \left[C_{6}\left(1+\left\|f_{1}+f_{2}\right\|^{12}\right) \exp \left(C_{6}\left\|f_{1}+f_{2}\right\|^{2}\right)\right] .
\end{aligned}
$$

Proof. From (2.5) and (2.6), we get

$$
\begin{aligned}
\left\|\nabla\left(\phi_{1}-\phi_{2}\right)\right\| \leq & \frac{1}{k}\left\|\nabla\left(\psi_{1}-\psi_{2}\right)\right\|+\frac{1}{k}\left\|\psi_{1}-\psi_{2}\right\|_{L^{4}}\left\|\nabla \psi_{2}\right\|_{L^{4}} \\
& +2\left\|\psi_{1}-\psi_{2}\right\|_{L^{4}}\left\|A_{1}\right\|_{L^{4}}+\left\|A_{1}-A_{2}\right\| .
\end{aligned}
$$

On the other hand, from (1.1) and (1.2), it follows that

$$
\begin{aligned}
& \frac{\eta}{2} \frac{d}{d t} \int_{\Omega}\left|\psi_{1}-\psi_{2}\right|^{2} d x+\frac{1}{k^{2}} \int_{\Omega}\left|\nabla\left(\psi_{1}-\psi_{2}\right)\right|^{2} d x \\
& \leq \eta k\left\|\phi_{1}-\phi_{2}\right\|\left\|\psi_{1}-\psi_{2}\right\|+\frac{2}{k}\left\|\psi_{1}-\psi_{2}\right\|\left\|A_{1}-A_{2}\right\|_{L^{4}}\left\|\nabla \psi_{1}\right\|_{L^{4}} \\
& +\frac{2}{k}\left\|\nabla\left(\psi_{1}-\psi_{2}\right)\right\|\left\|\psi_{1}-\psi_{2}\right\|_{L^{4}}\left\|A_{2}\right\|_{L^{4}}+\left\|\psi_{1}-\psi_{2}\right\|\left\|A_{1}-A_{2}\right\|_{L^{4}}\left\|A_{1}+A_{2}\right\|_{L^{4}}
\end{aligned}
$$

and

$$
\begin{aligned}
& \frac{1}{2} \frac{d}{d t} \int_{\Omega}\left(A_{1}-A_{2}\right)^{2} d x+\frac{1}{2} \int_{\Omega}\left|\operatorname{curl}\left(A_{1}-A_{2}\right)\right|^{2} d x \\
& \leq \frac{1}{k}\left\|\nabla\left(\psi_{1}-\psi_{2}\right)\right\|\left\|A_{1}-A_{2}\right\|+\frac{1}{k}\left\|\psi_{1}-\psi_{2}\right\|_{L^{4}}\left\|A_{1}-A_{2}\right\|\left\|\nabla \psi_{2}\right\|_{L^{4}} \\
& +2\left\|\psi_{1}-\psi_{2}\right\|_{L^{4}}\left\|A_{1}-A_{2}\right\|\left\|A_{1}\right\|_{L^{4}}+\left\|A_{1}-A_{2}\right\|^{2}+\frac{1}{2}\|h\|^{2}\left|f_{1}(t)-f_{2}(t)\right|^{2} .
\end{aligned}
$$

Using (2.8) and (2.9), one easily gets (2.13) from (2.14)-(2.16).

\section{Proof of Theorem 1.3}

With the help of the estimates in Section 2, we are able to complete the proof of Theorem 1.3 in this section. We begin with the following lemma:

Lemma 3.1. If $T>0$ is small enough, then there exists a positive constant $R$, such that $B$ maps $D$ into itself.

Proof.

$$
\begin{aligned}
& \left\|B f-\frac{g^{\prime}}{h_{0}}\right\|^{2}=\int_{0}^{T} h_{0}^{-2}\left\{\int_{\Omega}\left[A \Delta w+w \operatorname{Re}\left(\frac{i}{k} \nabla \psi+\psi A\right) \bar{\psi}\right] d x\right\}^{2} d t \\
& \leq \frac{2}{h^{2}(T)} \int_{0}^{T}\left(\|A\|^{2}\|\Delta w\|^{2}+\|w\|^{2}\left\|\frac{i}{k} \nabla \psi+\psi A\right\|^{2}\right) d t \\
& \leq \frac{2 T}{h^{2}(T)}\|\Delta w\|^{2}\left[\left\|A_{0}\right\|^{2}+2 C_{1}^{2}\left(|\Omega| T+\frac{\eta}{2}\left\|\psi_{0}\right\|^{2}\right)+4 R^{2}\|h\|^{2}+2\left\|\frac{g^{\prime}}{h_{0}}\right\|^{2}\|h\|^{2}\right]
\end{aligned}
$$


400 INVERSE PROBLEM OF GINZBURG-LANDAU EQUATIONS IN SUPERCONDUCTIVITY

$$
\begin{aligned}
& +\frac{2}{h^{2}(T)}\|w\|^{2}\left(|\Omega| T+\frac{\eta}{2}\left\|\psi_{0}\right\|^{2}\right) \\
& \leq R^{2}:=\frac{4 T}{h^{2}(T)}\|\Delta w\|^{2}\left[\left\|A_{0}\right\|^{2}+2 C_{1}^{2}\left(|\Omega| T+\frac{\eta}{2}\left\|\psi_{0}\right\|^{2}\right)+2\left\|\frac{g^{\prime}}{h_{0}}\right\|^{2}\|h\|^{2}\right] \\
& +\frac{4}{h^{2}(T)}\|w\|^{2}\left(|\Omega| T+\frac{\eta}{2}\left\|\psi_{0}\right\|^{2}\right)
\end{aligned}
$$

if

$$
\frac{8}{h^{2}(T)}\|\triangle w\|^{2}\|h\|_{L^{\infty}\left(0, T ; L^{2}(\Omega)\right)}^{2} T \leq \frac{1}{2}
$$

This proves the lemma.

LEMma 3.2. Let (H1)-(H5) and (3.1) be satisfied, then there exists a positive integer $k$, such that the operator $B^{k}$ is a contraction mapping in the ball $D$.

Proof. Let $f_{1}$ and $f_{2} \in D$, and $\left(\psi_{i}, A_{i}, \phi_{i}\right)(i=1,2)$ be the unique solution to the problem (1.1)-(1.4) with $f$ replaced by $f_{1}$ and $f_{2}$, respectively. From (2.13) and Lemma 3.1, we find that

$$
\begin{aligned}
& \left\|B f_{1}-B f_{2}\right\|_{L^{2}(0, t)}^{2}=\int_{0}^{t}\left|B f_{1}-B f_{2}\right|^{2} d \tau \\
& \leq \frac{2}{|h(T)|}\left(\|\Delta w\|^{2}+3\|w\|^{2}\right) \int_{0}^{t}\left\|A_{1}-A_{2}\right\|_{L^{\infty}\left(0, \tau ; L^{2}(\Omega)\right)}^{2} d \tau \\
& \quad+\frac{6}{|h(T)|}\left(\left\|\nabla\left(\psi_{1}+\psi_{2}\right)\right\|_{L^{\infty}\left(0, T ; L^{2}(\Omega)\right)}^{2}+\left\|A_{2}\right\|_{L^{\infty}\left(0, T ; L^{2}(\Omega)\right)}^{2}\right)\|w\|_{L^{\infty}(\Omega)}^{2} \\
& \times \int_{0}^{t}\left\|\psi_{1}-\psi_{2}\right\|_{L^{\infty}\left(0, \tau ; L^{2}(\Omega)\right)}^{2} d \tau \\
& \leq C_{7} \int_{0}^{t}\left|f_{1}-f_{2}\right|_{L^{2}(0, \tau)}^{2} d \tau,
\end{aligned}
$$

which leads to

$$
\left\|B f_{1}-B f_{2}\right\|_{L^{2}(0, t)} \leq\left(C_{7} \int_{0}^{t}\left\|f_{1}-f_{2}\right\|_{L^{2}(0, \tau)}^{2} d \tau\right)^{1 / 2} .
$$

By virtue of induction on $k$, the inequality (3.2) implies

$$
\left\|B^{k} f_{1}-B^{k} f_{2}\right\|_{L^{2}(0, T)} \leq\left(\frac{C_{7}^{k} T^{k}}{k !}\right)^{1 / 2}\left\|f_{1}-f_{2}\right\|_{L^{2}(0, T)} .
$$

Obviously, as $k \rightarrow \infty$,

$$
\frac{C_{7}^{k} T^{k}}{k !} \rightarrow 0
$$

and therefore, there exists a positive integer $k_{0}$ such that

$$
\left(\frac{C_{7}^{k_{0}} T^{k_{0}}}{k_{0} !}\right)^{1 / 2}<1
$$


Thus, by virtue of the estimate (3.3), the operator $B^{k_{0}}$ is a contracting mapping in the ball $D$. This proves Lemma 3.2 .

Finally, Theorem 1.3 follows easily from Lemmas 3.1 and 3.2, and the contraction mapping theorem. This completes the proof of Theorem 1.3.

Acknowledgements. The authors are indebted to Prof. Lishang Jiang and the referee for Remark 1.3 ii) is given by their suggestions.

\section{REFERENCES}

[1] Z. M. Chen, C. Elliott and Q. Tang, Justification of a two-dimensional evolutionary GinzburgLandau superconductivity model, RAIRO Model, Math. Anal. Numer., 32, 25-50, 1998.

[2] Z. M. Chen, K. H. Hoffmann and J. Liang, On a nonstationary Ginzburg-Landau superconductivity model, Math. Meth. Appl. Sci., 16, 855-875, 1993.

[3] Z. M. Chen and K. H. Hoffmann, Optimal control of dynamical Ginzburg-Landau vortices in superconductivity, Numer. Funct. Anal. Optimiz., 17, 241-258, 1996.

[4] Q. Du, Global existence and uniqueness of solutions of the time-dependent Ginzburg-Landau model for superconductivity, Appl. Anal., 521, 1-17, 1994.

[5] J. Fan, The long-time behavior of transient Ginzburg-Landau equation of superconductivity, Appl. Math. Lett., 9, 107-109, 1996.

[6] J. Fan and S. Jiang, Global existence of weak solutions of a time dependent 3-D GinzburgLandau model for superconductivity, Appl. Math. Lett., 16, 435-440, 2003.

[7] J. Fan and S. Jiang, The long time behavior of Ginzburg-Landau equation for superconductivity with Coulomb gauge, preprint 2004.

[8] P. L. Lions, Mathematical Topics in Fluid Mechanics, Volume II, Comperssible Models, Oxford Science Publications, Clarendon Press, Oxford, 1998.

[9] A. I. Prilepko, D. G. Orlovsky and I. A. Vasin, Methods for Solving Inverse Problems in Mathematical Physics, Marcel Dekker, Inc., New York, Basel, 1999.

[10] Q. Tang, On an evolutionary system of Ginzburg-Landau equations with fixed total magnetic flux, Comm. PDE, 20, 1-36, 1995.

[11] Q. Tang and S. Wang, Time dependent Ginzburg-Landau equation of superconductivity, Physica D, 88, 139-166, 1995 . 